\title{
COMPARAÇÃO ENTRE MÉTODOS PARA ESTIMAR A PRODUTIVIDADE DE GRÃOS DE MILHO ${ }^{1}$
}

\author{
Comparison between methods for grains yield estimation in corn field
}

\author{
Victor do Nascimento Rodrigues², Renzo Garcia Von Pinho ${ }^{3}$, Carlos Maurício Paglis ${ }^{3}$, \\ Júlio Sílvio de Sousa Bueno Filho ${ }^{4}$, André Humberto de Brito ${ }^{5}$
}

\begin{abstract}
RESUMO
O objetivo deste trabalho foi avaliar a eficiência de dois métodos utilizados para estimar a produtividade de grãos em lavouras comerciais de milho. Foram avaliados o método de Reetz, desenvolvido na Universidade de Illinois, nos Estados Unidos, e o método da Emater-MG, utilizado em concursos de produtividade. Analisou-se a eficiência dos métodos em três áreas, sendo uma na UFLA, uma na Fazenda Campo Lindo em Lavras-MG e uma na Fazenda Dutra em Itumirim-MG, obtendo-se as produtividades estimadas e as produtividades reais em cada local. Para obtenção das produtividades estimadas, as áreas foram divididas em 4 talhões, sendo colhida uma parcela por talhão. Cada parcela constituiu-se de 7 sub-amostras de $4 \mathrm{~m}^{2}$ com 3 espigas cada, distribuídas em 4 linhas de $10 \mathrm{~m}$. Para o método de Reetz, utilizou-se na expressão de cálculo o número de espigas em $4 \mathrm{~m}^{2}$, o número de fileiras de grãos e o número de grãos por fileira. Para o método da Emater, utilizou-se o espaçamento entre fileiras, número de espigas em 10 m e o peso médio de grãos por espiga. Com as estimativas das sub-amostras, foram feitas análises de variância para cada local e para cada método. Com os componentes da variância foram determinadas as funções desta, de acordo com o número de parcelas e sub-amostras para posterior determinação de intervalos de 95\% de confiança da produtividade média estimada pelos métodos, com o intuito de verificar se estes continham as produtividades reais dos locais. Concluise que o método da Emater é útil para comparações entre produtividades de diferentes áreas, podendo ser reduzido pela metade o número de espigas amostradas por parcela. Esse método tende a superestimar a produtividade real com o aumento do número de parcelas e sub-amostras, dado o aumento da precisão das estimativas. O método de Reetz precisa ser adaptado a grupos de híbridos com características semelhantes.
\end{abstract}

Termos para indexação: Zea mays, milho, estimativa de produção, produtividade de grãos.

\begin{abstract}
The objective of this work was to evaluate the efficiency of two methods used to estimate the productivity of grains on commercial corn farms. The Reetz method, developed at the University of Illinois, in the United States, and the Emater-MG method, used in productivity contests were appraised. The efficiency of the methods was analyzed in three areas, one being in the UFLA and one in Campo Lindo farm in Lavras-MG, and one in Dutra farm in Itumirim-MG. The productivity estimates and the real productivity being obtained in each local. For obtaining of the estimated productivities, the areas were divided in 4 plots, one portion being harvested per plot. Each portion was made up of 7 sub-samples of $4 \mathrm{~m}^{2}$ with 3 ears of corn each, distributed in 4 lines of $10 \mathrm{~m}$. For the Reetz method, the number of ears of corn in $4 \mathrm{~m}^{2}$, the number of rows of grain and the number of grains per row were used in the calculation expression. For the Emater method, the spacing among lines, number of ears of corn in $10 \mathrm{~m}$ and the average grain weight per ear of corn was used. With the estimates of the sub-samples, variance analysis were made for each local and for each method. With the variance components of each analysis, variance functions were determined in accordance with the number of portions and sub-samples for subsequent determination of intervals of $95 \%$ confidence of the estimated average productivity for the methods, with the intention of verifying if these contained the real productivities of the locals. It is concluded that the Emater method is useful for comparisons among productivities of different areas. The number of ears of corn sampled per portion that has been recommended by the Emater method can be reduced by half. The Emater method tends to overestimate the real productivity with the increase of the number of portions and sub-samples, given the increase of the precision of the estimates. The Reetz method needs to be adapted to hybrid groups with similar characteristics.
\end{abstract}

Index terms: Zea mays, corn, estimative of productivity, productivity of grains.

(Recebido para publicação em 27 de abril de 2004 e aprovado em 24 de novembro de 2004)

\footnotetext{
1. Parte da dissertação do primeiro autor para obtenção do Título de Mestre em Agronomia/Fitotecnia apresentado à Universidade Federal de Lavras/UFLA - Caixa Postal 3037 - 37.200-000 - Lavras, MG.

2. Engenheiro Agrônomo, M.Sc. Professor do Departamento de Agricultura/UFLA.

3. Engenheiro Agrônomo, Dr. Professor do Departamento de Agricultura/UFLA.

4. Engenheiro Agrônomo, Dr. Professor do Departamento de Ciências Exatas/UFLA.

5. Graduando em Agronomia, bolsista de iniciação científica PIBIICT/FAPEMIG.
} 


\section{INTRODUÇÃO}

Muitos agricultores procuram obter uma estimativa da produtividade antes da época da colheita, pois podem utilizar a previsão da produção para avaliar as necessidades futuras de transporte e armazenamento do produto, bem como os prováveis ganhos na sua comercialização. As estimativas de produtividade são úteis em comparações nos ensaios de híbridos/variedades, verificando a variabilidade de produção em uma mesma área ou entre diferentes áreas de cultivo, ou comparando diferentes práticas de manejo.

Embora a cultura tenha importância mundial, são poucos os métodos utilizados para estimar a produtividade de milho, pois esta é influenciada por inúmeros fatores. Em grande parte, os métodos são modelos matemáticos para simulação baseada em observações relacionadas à fisiologia e fenologia da planta e às condições meteorológicas do local. Da mesma maneira, existem poucos métodos práticos e simples para estimar o rendimento de grãos da cultura.

Um método que tem sido muito utilizado para estimar a produtividade de grãos de milho é o utilizado pela Emater-MG (EMATER-MG, 2000). Esse método é aplicado em diversas propriedades agrícolas participantes do concurso anual de produtividade de milho do estado, e baseia-se em componentes de produção da planta, exigindo a determinação da umidade e do peso médio dos grãos, espaçamento entre linhas e o número médio de espigas obtidos em várias amostragens. As lavouras participantes do concurso são divididas em 3 categorias. Nas áreas com 1 a 10 ha, com área mínima de 5 ha com um mesmo cultivar, a amostragem é realizada em 4 pontos, por meio de sorteio de 4 fileiras de 10 metros lineares por ponto e colheita de 80 espigas no total. Nas áreas com 10 a 50 ha e área mínima de 25 ha com um mesmo cultivar, a amostragem é realizada em 4 pontos, mas sorteando-se 8 fileiras de 10 metros lineares por ponto e colhendo-se 160 espigas no total. Nas áreas acima de 50 ha e área mínima de 50 ha com um mesmo cultivar, também são amostrados 4 pontos, sendo colhido 1 ha por ponto com uma colhedora mecânica e calculada a perda na colheita (EMATER-MG, 2000).

Outro método bem simples e pouco conhecido no Brasil, desenvolvido na Universidade de Illinois, nos Estados Unidos, é também baseado na coleta de espigas na pré-colheita sendo estimada a produtividade de grãos a partir da contagem do número de espigas, do número de fileiras de grãos e do número de grãos por fileira de cada espiga (REETZ, 1987). Nesse método, em vários pontos da lavoura com área de $4 \mathrm{~m}^{2}$, todas as espigas são contadas e somente três espigas são colhidas por ponto de amostragem. Em seguida, são determinados o número de fileiras de grãos e o número de grãos por fileira para cada espiga, sendo estes dados utilizados em uma expressão para se estimar a produtividade de grãos. O método não fixa o número de pontos amostrados e nem determina o número de pontos de acordo com a área da lavoura. A sua precisão será maior quando utilizado para comparações entre áreas distintas ou entre diferentes práticas de manejo, devendo-se ter atenção na seleção de locais representativos para a amostragem e, se necessário, aumentar o número de amostras na lavoura. Variações no peso e no tamanho dos grãos, características do híbrido, fatores de manejo e a ocorrência de condições ambientais inesperadas poderão afetar as comparações (REETZ, 1987).

Apesar do método da Emater e do método de Reetz serem conhecidos, existem poucas informações sobre a eficiência de cada um deles. Em relação ao método de Reetz especificamente, há a necessidade de sua avaliação principalmente nas condições brasileiras.

O objetivo deste trabalho foi avaliar a eficiência do método da Emater e do método de Reetz para estimar a produtividade de grãos em lavouras comerciais de milho.

\section{MATERIAL E MÉTODOS}

O trabalho foi realizado no ano agrícola 2002/2003, em uma área do Departamento de Agricultura da UFLA, e em duas lavouras comerciais, uma localizada em Lavras-MG, na Fazenda Campo Lindo, e outra em Itumirim-MG, na Fazenda Dutra. Nos três locais considerados, foram realizados levantamentos planialtimétricos com Estação Total para determinação da área total cultivada com milho. Foram utilizados três híbridos simples recomendados para plantio na região (TABELA 1).

Em todas as áreas, o solo foi preparado convencionalmente e a semeadura realizada na primeira semana de dezembro, utilizando-se semeadoras de discos de tração mecanizada. Todas as operações foram feitas com as semeadoras reguladas para o espaçamento de $0,80 \mathrm{~m}$ entre linhas. Por ocasião da semeadura, foram aplicados aproximadamente $400 \mathrm{~kg} \cdot \mathrm{ha}^{-1}$ da fórmula $8(\mathrm{~N}): 28$ $\left(\mathrm{P}_{2} \mathrm{O}_{5}\right): 16\left(\mathrm{~K}_{2} \mathrm{O}\right)$, com 0,5\% de $\mathrm{Zn}$. Quando as plantas atingiram de 4 a 6 folhas, realizou-se uma única adubação de cobertura com a aplicação de $300 \mathrm{~kg} \cdot \mathrm{ha}^{-1}$ da fórmula $30(\mathrm{~N})$ : $00\left(\mathrm{P}_{2} \mathrm{O}_{5}\right)$ : $20\left(\mathrm{~K}_{2} \mathrm{O}\right)$. Para o controle de plantas daninhas, após a semeadura foi aplicado o herbicida Primestra ${ }^{\circledR}$ (7,0 litros.ha $\left.{ }^{-1}\right)$ em pré-emergência e as pragas controladas de acordo com as necessidades. 
TABELA 1 - Local de semeadura e características dos híbridos de milho utilizados. UFLA, Lavras-MG, 2004.

\begin{tabular}{lllllc}
\hline \multicolumn{1}{c}{ Local } & Híbrido & Empresa & Ciclo & \multicolumn{1}{c}{ Tipo de grão } & Nível de tecnologia \\
\hline UFLA & A 2345 & Bayer & Precoce & Semi-dentado amarelo & Alto \\
Fazenda Campo Lindo & Dow 8420 & Dow & Precoce & Duro alaranjado & Alto \\
Fazenda Dutra & Fort & Syngenta & Precoce & Duro vermelho & Alto \\
\hline
\end{tabular}

Na área da UFLA, para obtenção das produtividades reais corrigidas para $15,5 \%$ de umidade e expressas em t.ha ${ }^{-1}$, a colheita foi realizada manualmente coletando-se as espigas empalhadas para depois serem debulhadas mecanicamente com o uso de um debulhador acoplado ao trator. Após essa operação, utilizando-se uma balança digital e um medidor de umidade portátil, foi obtida a produção real. Nas áreas localizadas nas Fazendas Campo Lindo e Dutra, as colheitas foram realizadas mecanicamente. Posteriormente, utilizando-se as pesagens e umidades obtidas por ocasião da comercialização dos grãos, determinou-se a produção real.

As produtividades nos três locais foram estimadas pelo método da Emater-MG (2000) e pelo método proposto por Reetz (1987). Na pré-colheita, foram feitas adaptações nas amostragens propostas nos métodos originais para possibilitar a análise estatística. Os três locais considerados foram divididos em 4 talhões, seguindo as recomendações da Emater-MG (2000). Em cada local, foram amostradas aleatoriamente 4 parcelas, sendo uma parcela por talhão. A parcela constituiu-se de 4 linhas de 10 metros, sendo dividida em 7 sub-amostras, cada uma com 5 metros de comprimento e aproximadamente $4 \mathrm{~m}^{2}$, com pequenas variações do comprimento da linha, de acordo o espaçamento. De cada sub-amostra, foram amostradas 3 espigas representativas, totalizando 21 espigas por parcela, e obtidos valores da produtividade estimada para cada método.

Pelo método de Reetz (1987), a produtividade estimada foi obtida da média das produtividades estimadas de cada uma das três espigas, provenientes de cada sub-amostra, utilizando a seguinte expressão:

Produtividade (t.ha ${ }^{-1}$ a 15,5\% de umidade) $=\mathrm{A} \mathrm{x}$ B x C x 0,01116 x 0,063

em que:

A: número de espigas em $4 \mathrm{~m}^{2}$;

B: número de fileiras de grãos da espiga;

C: número de grãos por fileira, utilizando uma média de 3 fileiras de grãos por espiga, desconsiderando os grãos menores que a metade de um grão normal, presentes na extremidade da espiga;

0,01116: fator de correção do método;

0,063: valor para transformação de bushels por acre (bu. $\mathrm{A}^{-1}$ ) para t.ha ${ }^{-1}$.

Pelo método da Emater-MG (2000), a produtividade estimada foi obtida pela seguinte expressão:

Produtividade (t.ha ${ }^{-1}$ a $15,5 \%$ de umidade) $=$ [ (NE x P)/EM ]/1000

em que:

NE: número médio de espigas em 10 metros lineares.

P: peso médio de grãos por espiga corrigido para $15,5 \%$ de umidade, obtido da média do peso de grãos das 3 espigas coletadas;

EM: espaçamento médio entre linhas.

Para verificar a existência de diferença entre os métodos, foram realizadas três análises de variância, uma para cada local, com as produtividades médias estimadas das sub-amostras.

Visando a comparação das produtividades estimadas entre os locais, foi feita uma análise envolvendo os três locais para cada método, usando modelos hierárquicos com o fator aleatório parcela dentro do fator fixo local. Constatando-se diferenças significativas entre os locais, as produtividades médias estimadas foram submetidas ao teste de Tukey a $5 \%$ de probabilidade.

Baseado nos resultados da análise de variância e do teste de Tukey, realizou-se uma análise de variância para cada local e métodos, para posterior determinação da variabilidade e dos intervalos de confiança das produtividades estimadas.

Com os componentes da variância obtidos pelo método prático de Hicks, descrito por Ramalho et al. (2000), foram determinadas funções de variância de cada método de acordo com o número de parcelas e subamostras, utilizando as esperanças dos quadrados médios obtidos nas análises de variância.

Os componentes de variância do fator parcela foram determinados por meio de sistemas de equações das esperanças dos quadrados médios, em que: 


$$
\begin{aligned}
& \text { QMparcela }=\hat{\sigma}_{e^{2}}+7 \hat{\sigma}_{p^{2}} \\
& \text { QMerro }=\hat{\sigma}_{e^{2}} \\
& \hat{\sigma}_{e^{2}}=(\text { QMparcela-QMerro }) / 7
\end{aligned}
$$

Após o cálculo dos componentes de variância do fator parcela para cada análise, as funções de variância dos métodos foram determinadas pela equação:

$$
\hat{\sigma}^{2}=\left(\hat{\sigma}_{e^{2}}+n \hat{\sigma}_{p^{2}}\right) /(n \cdot k)
$$

Em que:

$\hat{\sigma}_{2}$ : estimativa da variância da produtividade estimada para $n$ sub-amostras e $k$ parcelas;

$\hat{\sigma}_{e^{2}}$ : estimativa da variância do fator aleatório subamostra;

$\hat{\sigma}_{p^{2}}$ : estimativa da variância do fator aleatório parcela;

$n$ : número de sub-amostras dentro do fator aleatório parcela;

$k$ : número de parcelas.

Por meio dessa equação, as funções de variância foram representadas graficamente para observação da variabilidade das produtividades estimadas pelos métodos em função do aumento do número de parcelas e de subamostras. As variâncias obtidas foram então utilizadas para determinação dos intervalos de confiança (95\%) das produtividades médias estimadas, para verificar se os métodos determinam a produtividade real dos locais, de acordo com a seguinte fórmula, em que $n$ é o número de sub-amostras:

$$
\text { IC }_{(1-\alpha) \%}(\mu)=\mathrm{X} \pm t_{(\mathrm{n}-1, \alpha / 2)} . \hat{\sigma}
$$

As análises foram realizadas com a utilização dos programas Microsoft Excel $2000^{\circledR}$ e Sisvar $^{\circledR}$ (FERREIRA, 2000).

\section{RESULTADOS E DISCUSSÃO}

Foi observado efeito altamente significativo $(\mathrm{p} \leq 0,01)$ para a fonte de variação parcela nas áreas da UFLA e da Fazenda Campo Lindo. Houve diferença significativa $(\mathrm{p} \leq 0,05)$ entre os métodos somente na Fazenda Dutra. A precisão experimental avaliada pelo coeficiente de variação (C. V.) foi considerada boa, com valores de 20,18\% para a área da UFLA, 15,26\% para a Fazenda Campo Lindo e 11,73\% para a Fazenda Dutra (TABELA 2).

As diferenças significativas encontradas para a fonte de variação parcela nas áreas da UFLA e da Fazenda Campo Lindo, possivelmente ocorreram em função da variabilidade espacial do solo. A área da UFLA, principalmente, apresenta grande variação na cor do solo, propriedade relacionada com drenagem, matéria orgânica, forma e conteúdo de ferro, fixação de fósforo e fertilidade (RESENDE et al., 1997). Independente do método utilizado, isso pode dificultar a seleção de locais representativos para as amostragens e afetar as estimativas de produtividade dos métodos.

Visando constatar possíveis diferenças das produtividades estimadas entre os locais e considerando cada método separadamente, realizou-se uma análise de variância para cada método envolvendo os três locais.

TABELA 2 - Resumo das análises de variância da produtividade de milho estimada (t.ha ${ }^{-1}$ ) envolvendo os dois métodos em cada local. UFLA, Lavras-MG, 2004.

Q. M.

\begin{tabular}{lcccc}
\cline { 3 - 5 } \multicolumn{1}{c}{ F. V. } & G. L. & UFLA & Fazenda Campo Lindo & Fazenda Dutra \\
\hline Método & 1 & 0,49 & 0,38 & $27,48 *$ \\
Parcela & 3 & $15,30 * *$ & $7,38 * *$ & 1,33 \\
Erro & 51 & 1,97 & 1,30 & 0,92 \\
\hline C. V. (\%) & & 20,18 & 15,26 & 11,73 \\
\hline Média & & 6,962 & 7,480 & 8,182 \\
\hline
\end{tabular}

* e ** : significativo ao nível de 0,05 e 0,01 de probabilidade pelo teste $\mathrm{F}$. 
No método da Emater, foi observado efeito altamente significativo ( $\mathrm{p} \leq 0,01)$ para a fonte de variação parcela. Além disso, para esse método, houve diferença significativa ( $\mathrm{p} \leq 0,05)$ para a fonte de variação local, o que não ocorreu para o método de Reetz, que não detectou essa diferença de produtividade, não sendo, portanto, recomendado para comparações de produtividade entre locais onde são utilizados diferentes genótipos (TABELA 3).

De acordo com Reetz (1987), para comparação entre locais ou entre tratamentos como, por exemplo, práticas de manejo, o método tem maior precisão se for utilizado o mesmo híbrido. Vale ressaltar ainda, que esse método foi desenvolvido para aplicação em condições edafo-climáticas diferentes das brasileiras, utilizando cultivares uniformes (híbridos simples) desenvolvidos em tais condições e alto nível tecnológico.

O fator de correção do método de Reetz (0,01116), provavelmente não é apropriado a diferentes híbridos, podendo ser objeto de novos estudos que poderão gerar novos fatores, de acordo com as caracterís- ticas dos híbridos como, por exemplo, base genética, peso específico e dureza do grão. Quanto à sua utilização no Brasil, isso se torna relevante porque existe uma grande diversidade das condições de cultivo de milho e uma utilização expressiva de cultivares de diferentes bases genéticas, predominando o uso de híbridos duplos e triplos (PINHO, 2001).

Em relação ao método da Emater, como já mencionado, foi observada diferença significativa entre locais, confirmando as diferenças verificadas para a produtividade real (TABELA 4). Esse resultado é importante uma vez que, devido à sua utilização em concursos de produtividade, o método mostrou capacidade de detectar diferenças de produtividade entre as áreas de plantio. Assim, devido à pesagem e à determinação da umidade dos grãos, o método da Emater, quando comparado ao método de Reetz, parece ser menos afetado pelas características dos híbridos, como o tamanho e o peso específico dos grãos, podendo ser usado em áreas que utilizam diferentes híbridos e práticas de manejo.

TABELA 3 - Resumo da análise de variância da produtividade de milho estimada (t.ha ${ }^{-1}$ ) envolvendo os três locais para cada método avaliado. UFLA, Lavras-MG, 2004.

\begin{tabular}{lccc} 
& & \multicolumn{2}{c}{ Q. M. } \\
\cline { 3 - 4 } \multicolumn{1}{c}{ F. V. } & G. L. & Reetz & Emater \\
\hline Local & 2 & 1,4231 & 29,3037 * \\
Parcela (Local) & 9 & 1,7865 & 8,1137 ** \\
Erro & 72 & 1,6406 & 1,0958 \\
\hline C. V. (\%) & & 17,52 & 13,47 \\
\hline Média & & 7,312 & 7,771 \\
\hline
\end{tabular}

* e **: significativo ao nível de $5 \%$ e $1 \%$ de probabilidade pelo teste $\mathbf{F}$;

TABELA 4 - Valores médios para as produtividades estimadas (t.ha ${ }^{-1}$ ) pelos dois métodos avaliados e produtividades reais obtidas em cada local. UFLA, Lavras-MG, 2004.

\begin{tabular}{lccc}
\hline \multicolumn{1}{c}{ Local } & Produtividade & \multicolumn{2}{c}{ Produtividade estimada } \\
\cline { 3 - 4 } & real & Reetz & Emater \\
\hline UFLA & 4,764 & $7,056 \mathrm{~A} \mathrm{a}$ & 6,869 A c \\
Fazenda Campo Lindo & 6,450 & $7,397 \mathrm{~A} \mathrm{a}$ & $7,562 \mathrm{~A} \mathrm{~b}$ \\
Fazenda Dutra & 8,077 & $7,482 \mathrm{~B} \mathrm{a}$ & $8,883 \mathrm{~A} \mathrm{a}$ \\
\hline D. M. S. (5\%) & & 0,670 & 0,819 \\
\hline
\end{tabular}

Médias seguidas das mesmas letras, minúsculas na coluna e maiúsculas na linha, não diferem entre si pelo teste de Tukey e pelo teste $F$, respectivamente, ao nível de $5 \%$ de probabilidade.

Ciênc. agrotec., Lavras, v. 29, n. 1, p. 34-42, jan./fev. 2005 
A diferença observada na produtividade estimada pelo método da Emater entre locais, permitiu a realização de uma análise de variância para cada local separadamente, visando sua posterior avaliação por meio da variabilidade das estimativas (funções de variância) e de intervalos de confiança. Para o método de Reetz, foi determinada apenas uma equação, baseada nas esperanças dos quadrados médios da Tabela 3 , devido à ausên- cia de diferença significativa para as produtividades estimadas entre os locais. Pode-se verificar que, aumentando-se o número de sub-amostras, ocorre um decréscimo na variabilidade da produtividade estimada por ambos os métodos, no entanto, esse decréscimo é mais acentuado quando são utilizadas poucas sub-amostras, sendo observada a mesma tendência considerando o número de parcelas (FIGURAS 1A, 2A, 3A e 4A).
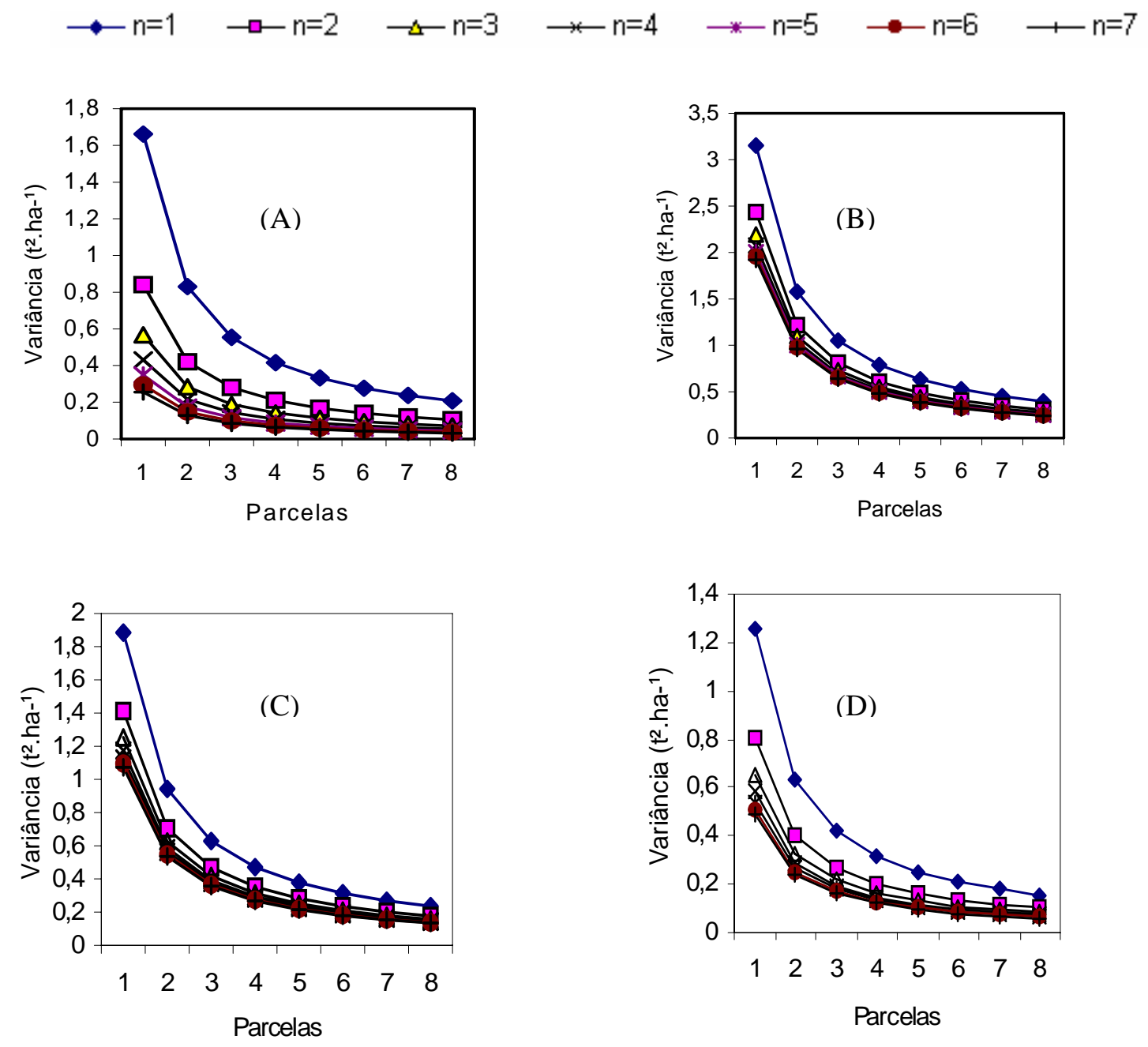

FIGURA 1 - Representação gráfica da variância da produtividade de milho estimada $\left(\mathrm{t}^{2}\right.$.ha $\left.{ }^{-1}\right)$ de acordo com o número $k$ de parcelas e o número $n$ de sub-amostras: (A) método de Reetz para os três locais; (B) método da Emater para a área da UFLA; (C) método da Emater para a área da Fazenda Campo Lindo; (D) método da Emater para a área da Fazenda Dutra. UFLA, Lavras-MG, 2004. 
Estes resultados indicam que quanto maior o número de espigas coletadas nos locais, menor é o erro da estimativa em relação à média das produtividades estimadas e maior é a precisão do método, já que haverá redução dos intervalos de confiança. A partir de aproximadamente metade do número total de sub-amostras, a redução na variância é insignificante, não provocando alterações significativas nos erros e nos intervalos de confiança dos métodos. Para o método da Emater, os resultados demonstram que o número de espigas coletadas por parcela pode ser reduzido à metade, sem alterações em sua precisão, facilitando e tornando mais rápido o processo de amostragem nas lavouras. Assim, podem ser coletadas 10 espigas por parcela em vez de 20, conforme é proposto pelo método (EMATER-MG, 2000).

Com 95\% de confiança, verificou-se que o método de Reetz determinou a produtividade real da área da UFLA (4,764 t.ha $\left.{ }^{-1}\right)$ somente quando consideradas uma parcela e uma sub-amostra. Na Fazenda Campo Lindo (6,450 t.ha $\left.{ }^{-1}\right)$, o método não funcionou a partir de 3 parcelas com 5 sub-amostras, 4 parcelas com 4 subamostras, 5 e 6 parcelas com 3 sub-amostras, 7 e 8 parcelas com 2 sub-amostras. Na Fazenda Dutra (8,077 t.ha ${ }^{-1}$ ), o método não funcionou a partir de 3 parcelas com 7 sub-amostras, 4 parcelas com 5 sub-amostras, 5 parcelas com 4 sub-amostras, 6, 7 e 8 parcelas com 3 sub-amostras (TABELA 5).

Para o método da Emater, foi observado que a validade das produtividades estimadas depende do número considerado de parcelas e sub-amostras. Com o aumento do número de espigas amostradas, ocorreu uma super-estimativa das produtividades reais. Para a área da UFLA, os intervalos de confiança não contêm a produtividade real $\left(4,764\right.$ t.ha $\left.^{-1}\right)$ a partir de 3 parcelas com 3 sub-amostras, 4 parcelas com 2 subamostras, 5, 6, 7 e 8 parcelas (TABELA 6). Para a área da Fazenda Campo Lindo, os intervalos de confiança não contêm a produtividade real $\left(6,450\right.$ t.ha $\left.^{-1}\right)$ quando se consideram 6 parcelas com mais de 3 subamostras e 7 e 8 parcelas com mais de 1 sub-amostra cada (TABELA 7). Para a área da Fazenda Dutra $\left(8,077\right.$ t.ha $\left.^{-1}\right)$, o mesmo ocorre quando se consideram 5 parcelas com mais de 4 sub-amostras, 6 parcelas com mais de 3 sub-amostras, 7 parcelas com mais de 2 sub-amostras e 8 parcelas com mais de 1 subamostra (TABELA 8).

TABELA 5 - Intervalos de 95\% de confiança para a média das produtividades estimadas (t.ha ${ }^{-1}$ ) pelo método de Reetz para os três locais, de acordo com o número $k$ de parcelas e o número $n$ de sub-amostras. UFLA, Lavras-MG, 2004.

\begin{tabular}{|c|c|c|c|c|c|c|c|c|c|c|c|c|c|c|c|c|}
\hline \multirow{3}{*}{$\begin{array}{c}\text { Sub- } \\
\text { amostras } \\
(n)\end{array}$} & \multicolumn{16}{|c|}{ Parcelas (k) } \\
\hline & \multicolumn{2}{|c|}{1} & \multicolumn{2}{|l|}{2} & \multicolumn{2}{|l|}{3} & \multicolumn{2}{|l|}{4} & \multicolumn{2}{|c|}{5} & \multicolumn{2}{|c|}{6} & \multicolumn{2}{|c|}{7} & \multicolumn{2}{|c|}{8} \\
\hline & L. I. & L. S. & L. I. & L. S. & L. I. & L. S. & L. I. & L.S. & L. I. & L. S. & L. I. & L. S. & L. I. & L. S. & L. I. & L. S. \\
\hline 1 & 4,158 & 10,466 & 5,082 & 9,542 & 5,491 & 9,133 & 5,735 & 8,889 & 5,901 & 8,723 & 6,024 & 8,600 & 6,120 & 8,504 & 6,197 & 8,427 \\
\hline 2 & 5,068 & 9,556 & 5,725 & 8,899 & 6,016 & 8,608 & 6,190 & 8,434 & 6,308 & 8,316 & 6,396 & 8,228 & 6,464 & 8,160 & 6,519 & 8,105 \\
\hline 3 & 5,468 & 9,156 & 6,008 & 8,616 & 6,248 & 8,376 & 6,390 & 8,234 & 6,487 & 8,137 & 6,559 & 8,065 & 6,615 & 8,009 & 6,660 & 7,964 \\
\hline 4 & 5,706 & 8,918 & 6,176 & 8,448 & 6,385 & 8,239 & 6,509 & 8,115 & 6,594 & 8,030 & 6,656 & 7,968 & 6,705 & 7,919 & 6,744 & 7,880 \\
\hline 5 & 5,867 & 8,757 & 6,290 & 8,334 & 6,477 & 8,147 & 6,589 & 8,035 & 6,666 & 7,958 & 6,722 & 7,902 & 6,766 & 7,858 & 6,801 & 7,823 \\
\hline 6 & 5,985 & 8,639 & 6,373 & 8,251 & 6,546 & 8,078 & 6,648 & 7,976 & 6,718 & 7,906 & 6,770 & 7,854 & 6,810 & 7,814 & 6,843 & 7,781 \\
\hline 7 & 6,076 & 8,548 & 6,438 & 8,186 & 6,598 & 8,026 & 6,694 & 7,930 & 6,759 & 7,865 & 6,807 & 7,817 & 6,845 & 7,779 & 6,875 & 7,749 \\
\hline
\end{tabular}

Ciênc. agrotec., Lavras, v. 29, n. 1, p. 34-42, jan./fev. 2005 
TABELA 6 - Intervalos de 95\% de confiança para a média das produtividades estimadas (t.ha ${ }^{-1}$ ) pelo método da Emater para a área da UFLA, de acordo com o número $k$ de parcelas e o número $n$ de sub-amostras. UFLA, Lavras-MG, 2004.

\begin{tabular}{|c|c|c|c|c|c|c|c|c|c|c|c|c|c|c|c|c|}
\hline \multirow{3}{*}{$\begin{array}{c}\text { Sub- } \\
\text { amostras } \\
(n)\end{array}$} & \multicolumn{16}{|c|}{ Parcelas (k) } \\
\hline & \multicolumn{2}{|c|}{1} & \multicolumn{2}{|c|}{2} & \multicolumn{2}{|c|}{3} & \multicolumn{2}{|c|}{4} & \multicolumn{2}{|l|}{5} & \multicolumn{2}{|l|}{6} & \multicolumn{2}{|l|}{7} & \multicolumn{2}{|c|}{8} \\
\hline & L. I. & L. S. & L. I. & L. S. & L. I. & L. S. & L. I. & L. S. & L. I. & L. S. & L. I. & L. S. & L. I. & L. S. & L. I. & L. S. \\
\hline 1 & 2,524 & 11,214 & 3,796 & 9,942 & 4,360 & 9,378 & 4,696 & 9,042 & 4,926 & 8,812 & 5,095 & 8,643 & 5,227 & 8,511 & 5,333 & 8,405 \\
\hline 2 & 3,051 & 10,687 & 4,169 & 9,569 & 4,665 & 9,073 & 4,960 & 8,778 & 5,162 & 8,576 & 5,310 & 8,428 & 5,426 & 8,312 & 5,519 & 8,219 \\
\hline 3 & 3,244 & 10,494 & 4,306 & 9,432 & 4,776 & 8,962 & 5,056 & 8,682 & 5,248 & 8,490 & 5,389 & 8,349 & 5,499 & 8,239 & 5,587 & 8,151 \\
\hline 4 & 3,344 & 10,394 & 4,377 & 9,361 & 4,834 & 8,904 & 5,107 & 8,631 & 5,293 & 8,445 & 5,430 & 8,308 & 5,537 & 8,201 & 5,623 & 8,115 \\
\hline 5 & 3,406 & 10,332 & 4,420 & 9,318 & 4,869 & 8,869 & 5,137 & 8,601 & 5,320 & 8,418 & 5,455 & 8,283 & 5,560 & 8,178 & 5,645 & 8,093 \\
\hline 6 & 3,447 & 10,291 & 4,450 & 9,288 & 4,894 & 8,844 & 5,158 & 8,580 & 5,339 & 8,399 & 5,472 & 8,266 & 5,576 & 8,162 & 5,659 & 8,079 \\
\hline 7 & 3,477 & 10,261 & 4,471 & 9,267 & 4,911 & 8,827 & 5,173 & 8,565 & 5,352 & 8,386 & 5,484 & 8,254 & 5,587 & 8,151 & 5,670 & 8,068 \\
\hline
\end{tabular}

TABELA 7 - Intervalos de 95\% de confiança para a média das produtividades estimadas (t.ha ${ }^{-1}$ ) pelo método da Emater para a área da Fazenda Campo Lindo, de acordo com o número $k$ de parcelas e o número $n$ de sub-amostras. UFLA, Lavras-MG, 2004.

\begin{tabular}{|c|c|c|c|c|c|c|c|c|c|c|c|c|c|c|c|c|}
\hline \multirow{3}{*}{$\begin{array}{c}\text { Sub- } \\
\text { amostras } \\
(n)\end{array}$} & \multicolumn{16}{|c|}{ Parcelas (k) } \\
\hline & \multicolumn{2}{|c|}{1} & \multicolumn{2}{|c|}{2} & \multicolumn{2}{|c|}{3} & \multicolumn{2}{|c|}{4} & \multicolumn{2}{|c|}{5} & \multicolumn{2}{|c|}{6} & \multicolumn{2}{|c|}{7} & \multicolumn{2}{|c|}{8} \\
\hline & L. I. & L. S. & L. I. & L. S. & L. I. & L. S. & L. I. & L. S. & L. I. & L. S. & L. I. & L. S. & L. I. & L. S. & L. I. & L. S. \\
\hline 1 & 4,202 & 10,922 & 5,186 & 9,938 & 5,623 & 9,501 & 5,883 & 9,241 & 6,060 & 9,064 & 6,191 & 8,933 & 6,293 & 8,831 & 6,373 & 8,751 \\
\hline 2 & 4,655 & 10,469 & 5,507 & 9,617 & 5,884 & 9,240 & 6,108 & 9,016 & 6,263 & 8,861 & 6,376 & 8,748 & 6,462 & 8,662 & 6,535 & 8,589 \\
\hline 3 & 4,823 & 10,301 & 5,626 & 9,498 & 5,980 & 9,144 & 6,193 & 8,931 & 6,336 & 8,788 & 6,443 & 8,681 & 6,527 & 8,597 & 6,592 & 8,532 \\
\hline 4 & 4,911 & 10,213 & 5,687 & 9,437 & 6,032 & 9,092 & 6,237 & 8,887 & 6,376 & 8,748 & 6,479 & 8,645 & 6,559 & 8,565 & 6,624 & 8,500 \\
\hline 5 & 4,965 & 10,159 & 5,726 & 9,398 & 6,064 & 9,060 & 6,263 & 8,861 & 6,401 & 8,723 & 6,501 & 8,623 & 6,580 & 8,544 & 6,643 & 8,481 \\
\hline 6 & 5,001 & 10,123 & 5,752 & 9,372 & 6,084 & 9,040 & 6,281 & 8,843 & 6,417 & 8,707 & 6,518 & 8,606 & 6,596 & 8,528 & 6,656 & 8,468 \\
\hline 7 & 5,028 & 10,096 & 5,771 & 9,353 & 6,100 & 9,024 & 6,295 & 8,829 & 6,430 & 8,694 & 6,527 & 8,597 & 6,605 & 8,519 & 6,666 & 8,458 \\
\hline
\end{tabular}

TABELA 8 - Intervalos de 95\% de confiança para a média das produtividades estimadas (t.ha ${ }^{-1}$ ) pelo método da Emater para a área da Fazenda Dutra, de acordo com o número $k$ de parcelas e o número $n$ de sub-amostras. UFLA, Lavras-MG, 2004.

\begin{tabular}{|c|c|c|c|c|c|c|c|c|c|c|c|c|c|c|c|c|}
\hline \multirow{3}{*}{$\begin{array}{c}\text { Sub- } \\
\text { amostras } \\
(n)\end{array}$} & \multicolumn{16}{|c|}{ Parcelas (k) } \\
\hline & \multicolumn{2}{|r|}{1} & \multicolumn{2}{|r|}{2} & \multicolumn{2}{|r|}{3} & \multicolumn{2}{|c|}{4} & \multicolumn{2}{|c|}{5} & \multicolumn{2}{|r|}{6} & \multicolumn{2}{|r|}{7} & \multicolumn{2}{|c|}{8} \\
\hline & L. I. & L. S. & L. I. & L. S. & L. I. & L. S. & L. I. & L. S. & L. I. & L. S. & L. I. & L. S. & L. I. & L. S. & L. I. & L. S. \\
\hline 1 & 6,140 & 11,626 & 6,944 & 10,822 & 7,299 & 10,467 & 7,512 & 10,254 & 7,657 & 10,109 & 7,764 & 10,002 & 7,845 & 9,921 & 7,913 & 9,853 \\
\hline 2 & 6,686 & 11,080 & 7,330 & 10,436 & 7,614 & 10,152 & 7,783 & 9,983 & 7,901 & 9,865 & 7,987 & 9,779 & 8,053 & 9,713 & 8,105 & 9,661 \\
\hline 3 & 6,901 & 10,865 & 7,482 & 10,284 & 7,738 & 10,028 & 7,892 & 9,874 & 7,997 & 9,769 & 8,075 & 9,691 & 8,133 & 9,633 & 8,182 & 9,584 \\
\hline 4 & 7,018 & 10,748 & 7,565 & 10,201 & 7,805 & 9,961 & 7,951 & 9,815 & 8,050 & 9,716 & 8,121 & 9,645 & 8,178 & 9,588 & 8,222 & 9,544 \\
\hline 5 & 7,092 & 10,674 & 7,616 & 10,150 & 7,848 & 9,918 & 7,987 & 9,779 & 8,083 & 9,683 & 8,153 & 9,613 & 8,204 & 9,562 & 8,250 & 9,516 \\
\hline 6 & 7,142 & 10,624 & 7,652 & 10,114 & 7,877 & 9,889 & 8,014 & 9,752 & 8,105 & 9,661 & 8,174 & 9,592 & 8,226 & 9,540 & 8,269 & 9,497 \\
\hline 7 & 7,181 & 10,585 & 7,679 & 10,087 & 7,901 & 9,865 & 8,032 & 9,734 & 8,121 & 9,645 & 8,187 & 9,579 & 8,240 & 9,526 & 8,279 & 9,487 \\
\hline
\end{tabular}




\section{CONCLUSÕES}

O método da Emater é útil para comparações entre produtividades de diferentes áreas, podendo ser reduzido pela metade o número de espigas amostradas por parcela.

O método da Emater tende a superestimar a produtividade real com o aumento do número de parcelas e subamostras, dado o aumento da precisão das estimativas.

O método de Reetz precisa ser adaptado a grupos de híbridos com características semelhantes.

\section{REFERÊNCIAS BIBLIOGRÁFICAS}

EMPRESA DE ASSISTÊNCIA TÉCNICA E EXTENSÃO RURAL DE MINAS GERAIS. Regulamento do concurso estadual de produtividade de milho ano 2000/2001. Belo Horizonte, 2000. 11 p.

FERREIRA, D. F. Análises estatísticas por meio do SISVAR para Windows versão 4.0. In: REUNIÃO ANUAL DA REGIÃO BRASILEIRA DA
SOCIEDADE INTERNACIONAL DE BIOMETRIA, 45., 2000, São Carlos. Resumos... São Carlos: UFSCAR, 2000. p. 35.

PINHO, R. G. von. Produção de milho no Brasil e no mundo: realidade e perspectivas. In: SIMPÓSIO DE ATUALIZAÇÃO EM GENÉTICA E MELHORAMENTO DE PLANTAS, 5., 2001, Lavras. Palestras... Lavras: UFLA, 2001. p. 3-13.

RAMALHO, M. A. P.; FERREIRA, D. F.; OLIVEIRA, A. C. de. Componentes da variância. In: Experimentação em genética e melhoramento de plantas. Lavras: UFLA, 2000. cap. 5, p. 69-86.

REETZ, H. Here's how to estimate yields for corn and soybeans before harvest. Better Crops With Plant Food, Atlanta, v. 71, p. 18-19, 1987.

RESENDE, M. et al. Interpretação. In: Pedologia: base para distinção de ambientes. 2. ed. Viçosa: NEPUT, 1997. cap. 2, p. 13-82. 